Proceedings of the Second Annual Forestry Symposium 1996: Management and Sustainable Utilization of Forest Resources, Sri Lanka, 6-7 December 1996. (Eds. Amarasekera, HS, Ranasinghe, D M S HK and Finlayson, W. Published by Department of Forestry and Environmental Science, University of Sri Jayewardenepura, Sri Lanka (1998)

\title{
SURVEY OF THE BUTTERFLY FAUNA OF UDAWALAWA NATIONAL PARK
}

\author{
M.D.P. Samarasinghe, P. Paranagama and S. Veediyabandara \\ National Herbarium, Royal Botanic Gardens, Peradeniya.
}

\begin{abstract}
The survey found 136 species, which are listed by families with their Latin and English names. They include 16 endemic, 45 threatened, 20 rare, and 3 very rare species. Some of their habitat preferences are briefly discussed, and the importance of the park butterfly fauna in relation to that of Sri Lanka as a whole is made clear.
\end{abstract}

\section{Introduction}

\section{Description of the area}

The Udawalawa National Park was established on 30th July 1972. It is in the lower catchment of the Udawalawa Resenoir in the low-country intermediate rainfall zone. On the right bank of the Walawe Ganga it is in Ratnapura District of the Sabaragamuwa Province. and on the left bank in Moneragala District of the Uva Province. The park lies between about $6^{\circ} 25^{\prime}$ to $6^{\circ} 35^{\prime} \mathrm{N}$ and $80^{\circ} 45^{\prime}$ to $81^{\circ} 0^{\prime} \mathrm{E}$ (Fig. 1).

The land area of the park is about 28,910 ha. The Udawalawa reservoir is included; its area when full is 3405 ha. The total area within the declared boundary of the park is therefore 32.315 ha.

\section{Environmental factors}

The climate is characterized by seasonal rainfall and uniformly high temperature.

The mean annual rainfall is about $1500 \mathrm{~mm}$ in the south. and gradually increases towards the north. There are two rainfall peaks, in April-May and October-November. A short dry spell comes in Febnuary-March, and there is a prolonged dry period from mid-May to the end of September. The main rainy season begins with convectional thunder storms in SeptemberOctober, which are followed by monsoon rains in November to mid-January. The April-May rainfall (the yala rains) is entirely due to convectional activity.

The mean anmual temperature is about $32^{\circ} \mathrm{C}$, and is fairly uniform throughout the year: there are no extreme fluctuations and the difference between the means for the hot season and the cool season months is less than $5^{\circ}$. The mean monthly maximum temperature varies from 30.0 to $37.2^{\circ}$ and the mean monthly minimum from 17.7 to $30^{\circ}$. 
The relative humidity at 8.30 hours varies from 61 to 94 and at 15.30 hours from 31 to $95 \%$. The wind speeds recorded at Angunakolapelessa, the nearest meteorological station to the park, are relatively low in the rainy seasons. In May, June and July they are high. In March and April and again during the north-east monsoon (November-January) they are relatively low. Throughout the year the winds speeds in the park are lower than they are near the south coast. which is not very far away.

The vegetation includes both grassland and different stages of forest succession. The forest has largely been cleared for shifting cultivation (chena), leading to anthropogenic grassland and scrub communities. Ten major land-use types were observed during this study. They are shown on the map in Fig. 2, and Table 1 gives their areas.

Although most of the vegetation in the park is secondary, it nevertheless supports an extensive range of fauna: 21 species of fishes, 12 amphibians, 18 snakes, 15 tetrapod reptiles, at least 184 birds and 39 mammals. This are high numbers in relation to the total of 108 species of fishes, 48 amphibians, 87 snakes, 75 tetrapod reptiles, 419 birds, and 89 mammals recorded in Sri Lanka. That it has 23 endemic and 48 threatened vertebrate species (IUCN, 1995) shows its importance for the biodiversity of the country. Six of the species are listed in the IUCN Red Data book as globally threatened.

Table 1 :Area of major habitat types

\begin{tabular}{lclc}
\hline \multicolumn{1}{c}{ Land-use type } & Area (ha) & Land-use type & Area (ha) \\
\hline I- Forest & 5227 & $\begin{array}{l}\text { 6-Scrub with } \\
\text { grassland } \\
7-\text { Medium height } \\
\text { scrub }\end{array}$ & 3718 \\
$\begin{array}{l}\text { 2- Secondary forest with } \\
\text { closed canopy }\end{array}$ & 2216 & 1683 \\
$\begin{array}{l}3-\text { Degraded/ } \\
\text { secondary open forest }\end{array}$ & 1949 & 3719 \\
$\begin{array}{l}4-\text { Highly grazed } \\
\text { grassland/ } \\
\text { abandoned chena }\end{array}$ & 1486 & $10-$ Teak plantation & 1076 \\
$\begin{array}{l}5-\text { Guinea } \\
\text { grass/savannah }\end{array}$ & 7662 & $\begin{array}{l}11-\text { Abandoned } \\
\text { paddy land }\end{array}$ & 174 \\
\hline
\end{tabular}




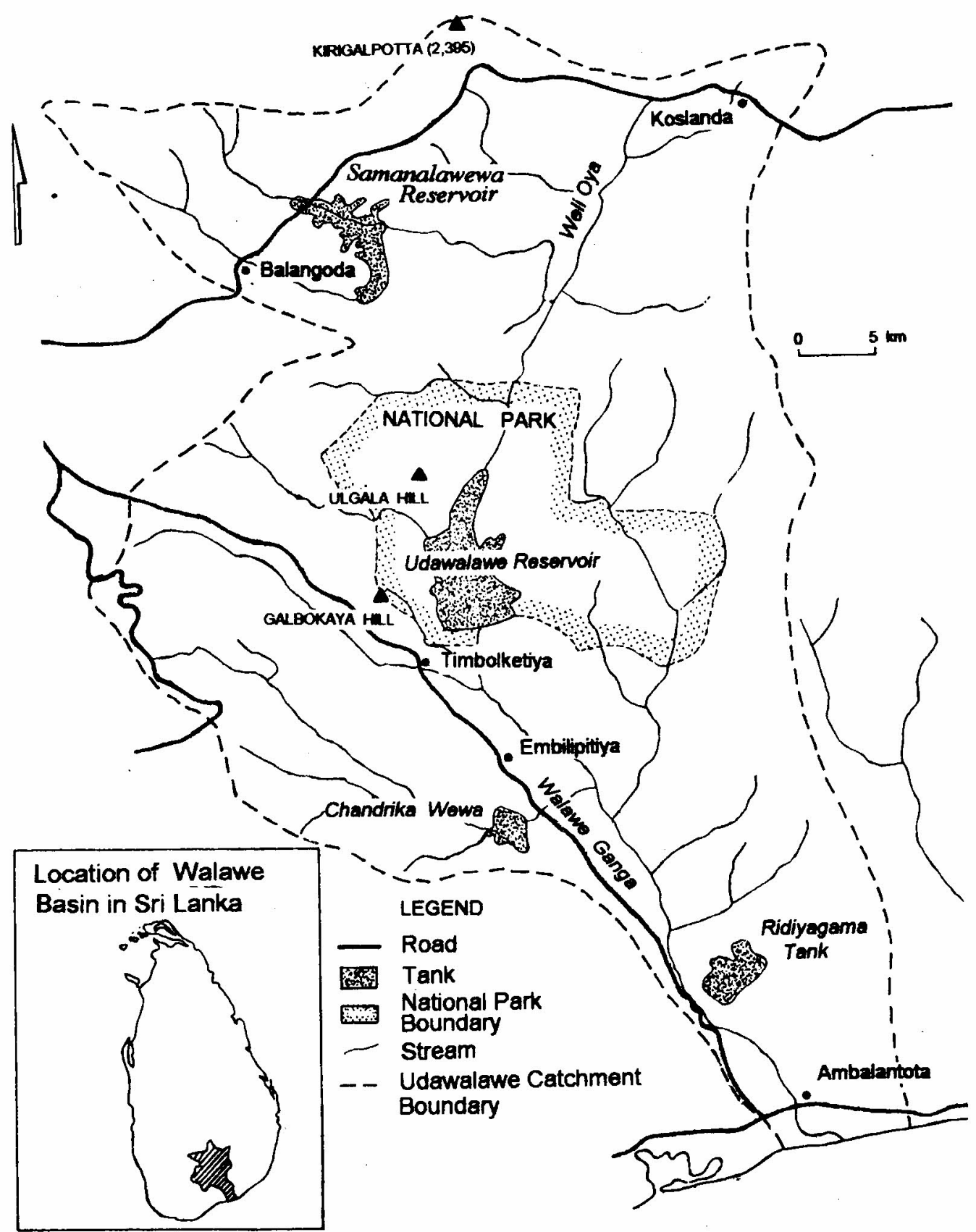

Figure 1: The location map of Udawalawe National Park 


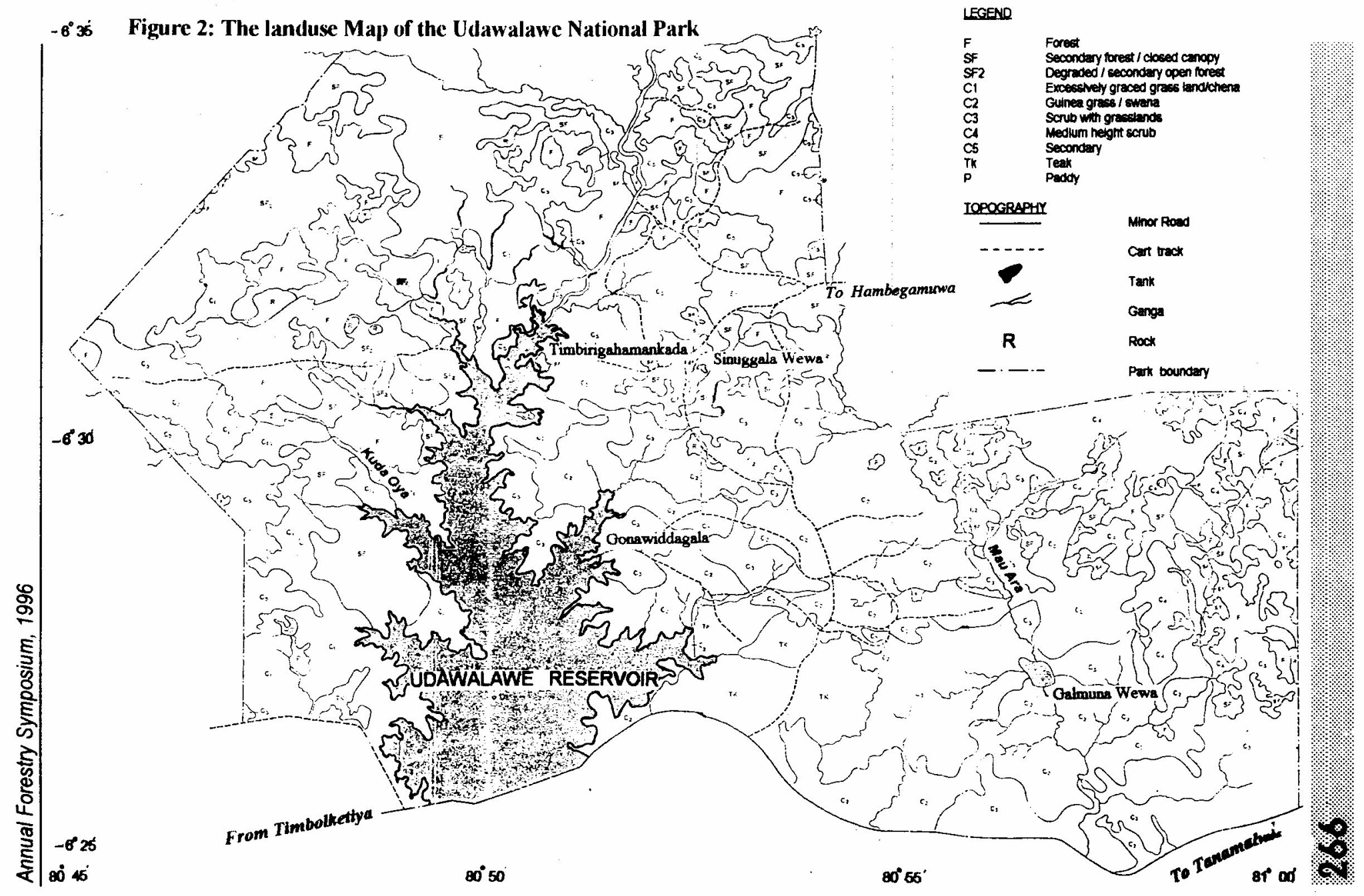




\section{Methods}

In January to August 1995 a ficld survey was carried out to identify the butterfly fauna of the park. Observations were made in each of the ten habitat types. Data were collected in daylight hours in a total of 20 days.

Butterflies were captured with a home made net (small-mesh type, $50 \mathrm{~cm}$ diameter, $60 \mathrm{~cm}$ decp, and 1-m handle). Collections were made along 100-m line transects in each habitat. Preserved specimens were deposited at the Department of Agricultural Biology. University of Peradeniya.

\section{Results and discussion}

According to Woodhouse (195() 242 butterflies belonging to 9 families have been recorded from Sri Lanka (Table 2). Among them there are 42 endemic, 11 very rare and 41 rare species. The IUCN considers that they include 81 threatened species and 4 red-listed threatened species (Table 4).

The present study shows that a total of 136 species of butterflies belonging to 9 families have been recorded in the park. The number of species observed in each family is given in Table 3. Among them, there are 16 endemic, 45 threatened, 20 rare, and 3 very rare species (Table 5).

\section{Table 2 : Butterfly fauna of Sri Lanka}

\begin{tabular}{lc} 
Families & Species \\
Danaidac & 12 \\
Satyridae & 16 \\
Amathusiidae & 1 \\
Bymphalidae & 37 \\
Erycinidac & 3 \\
Lycaenidae & 81 \\
Pieridae & 29 \\
Papilionidae & 15 \\
Hesperiidae & 48 \\
Tolal & 242 \\
\hline
\end{tabular}

The dominant land-use types in the park belong to categories 4, 5, 6, 7 and 10 of Table 1 . These represent habitats with much open grassland and scrub vegetation. The dominant butterfly family is therefore the Lycaenidae. Their dominant plant species are Lantana camara (Verbenaceae), (hromolaena odorata (Asteraceae). Tephrosia purpurea (Fabaceae), and grasses. These are the prefered food plants of the Lycaenidae. Some of this and other families. such as Papilio demoleus (lime butterlly), Eurema hecabe simulata (common grass yllow), Danaus chrysippus (plain tiger), Euploea coreasela (common Indian crow butterfly) are also found here because they prefer to live in open habitats. 
Annual Forestry Symposium, 1996

\begin{tabular}{lc}
\hline Table 3 : Butterfly fauna of Udawalawa National Park & \\
\hline Families & Species \\
\hline Danaidae & 4 \\
Satyridae & 6 \\
Amathusiidae & 1 \\
Nymphalidae & 23 \\
Erycinidae & 2 \\
Lycaenidae & 45 \\
Pieridae & 17 \\
Papilionidae & 7 \\
Hesperiidae & 31 \\
Total & 136 \\
\hline
\end{tabular}

Table 4: Status of the butterfly fauna of Sri Lanka

\begin{tabular}{lc}
\hline Fanilies & 9 \\
\hline Species & 242 \\
Endemic & 42 \\
Very rare & 11 \\
Rare & 41 \\
Threatened species & 81 \\
IUCN red list of threatened species & 4 \\
\hline
\end{tabular}

Table 5 : Status of the butterfly fauna of Udawalawa National Park

\begin{tabular}{lc} 
Families & 8 \\
Species & 136 \\
Endemic & 16 \\
Very rare & 3 \\
Rare & 20 \\
Threatened species & 43 \\
IUCN red list of threatened species & 2 \\
\hline
\end{tabular}

The butterflies belonging to the family Papilonidae very much prefer to live in canopy layer of the forests. Therefore the species Papilio polymnestor parinda (bluc mormon), Troides helena darsius (common bird-wing) and Papilio crino (common banded peacock butterfly) were more dominant in land-use categories 1, 2, 3 and 8 .

The commonest species was Euploea coreasela (the common Indian crow butterfly), and the next commonest were Danaus chrysippus (plain tiger). Ypthima ceylonica (white four-ring), Orsotriaena medus mandata (nigger), Neptis hylas varmon (common sailor), Jamides celenotissama (common cerulean), and Leptosia nina nina (Psyche). Species observed in all the main land-use types were Appias albina darada (common albatross butterfly), Eurema hecabe simulata (common gross yellow), and Papilio demoleus (lime butterfly). 
The butterfly population of the country has decreased to a considerable extent because of the use of agro-chemicals, mainly pesticides. It is now very hard to see a single butterfly in paddy land. Only a very few studies have been made of this attractive "super family" of the animal kingdom. It is very important to conserve their habitats and to make further studies of them. It is strongly recommended that the wildlife authorities should encourage visitors, both local and foreign, to practice butterfly watching, as they already practice bird watching in the National Parks, as an important eco-tourism activity.

\section{Acknowledgements}

The authors would like to thank for the staff of the Udawalawa National Park, The March for Conservation. the University of Colombo, and Professor H.P.N. Wijayagunasekera, Head of the Department of Agri-cultural Biology, University of Peradeniya, for their generous support.

IUCN (1995)

\section{References}

Woodhouse, L.G.O. (1950). The butterfly fauna of Ceylon.

\section{Appendix - Systematic list of butterfly fauna of Udawalawa National Park}

$\begin{array}{ll}\text { Kingdom } & \text { - Animalia } \\ \text { Phylum } & \text { - Arthropoda } \\ \text { Class } & \text { - Insecta } \\ \text { Order } & \text { - Lepidoptera } \\ \text { Sub-order } & \text { - Heteroneura } \\ \text { Super family } & \text { - Papilionoidea }\end{array}$

\section{Family Danaidae}

1. Idea lynceus jasonia Wd. (Ceylon tree nymph) ${ }^{\text {En Th }}$

2. Danaus aglea aglea $\mathrm{Cr}$. (glassy tiger)

3. Danaus chrysippus L.(plain tiger)

4. Euploea core asela M. (common Indian crow)

\section{Family Satyridac}

5. Mycalesis perseus typhlus Fr. (common bushbrown)

6. Lathe rohria nilgiriansis Guer. (common treebrown)

7. Ypthima cevlonica Hew. (white four-ring)

8. Orsotriaena medus mandata $\mathrm{M}$. (nigger)

9. Melanitis leda ismene $\mathrm{Cr}$. (common evening brown)

10. Elymnias hypermnestra fraterna But. (common palm fly) $)^{\text {En }}$

\section{Family Amathusiidae}

11. Discophora lepida ceylonica $\mathrm{Fr}$. (southern rajah) ${ }^{\mathrm{T}_{\mathrm{H}}}$

\section{Family Nymphalidae}

12. Charaxes polyxena psaphon Wd. (tawny rajah) ${ }^{\mathrm{T}_{\mathrm{h}}}$ 
13. Murwareda athamas madens Roth. (common nawab) ${ }^{\text {Th }}$

14. Apatura parisatis camiba M. (black prince)

15. Euthalia aconthea vasanta M. (baron)

16. Parthenos sylvia cyaneus M. (clipper) ${ }^{\text {En }}$ th

17. Limenitis procris $\mathrm{M}$. (commander) ${ }^{\mathrm{Enh}_{\mathrm{h}} \mathrm{Th}}$

18. Neptis jumbah Fr. (chestnut-streaked sailor)

19. Neptis hylas varmona $\mathrm{M}$. (common sailor)

20. Hypolimnas misippus L. (Danaid eggfly)

21. Doleschallia hylas vanmora M. (common sailor)

22. Nallima philanchus Wd. (blue oak leaf ${ }^{\mathrm{E}_{21}, \mathrm{Th}}$

23. Precis lintingensis $\mathrm{F}$. (yellow pansy)

24. Percis almana almana L. (pcacock pansy)

25. Vanessa cardui L. (painted lady)

26. Angvnnis hyperbius taprobana $\mathrm{M}$. (Indian fritillary)

27. ('upha ervmanthis placida $\mathrm{M}$. (rustic)

28. Phalanta phalanta Dry. (common leopard)

29. Vindula erota asela M. (cniser) ${ }^{\mathrm{En}}$. Th

30. (irroocharoa thais lanka M. (Tamil yeoman)

31. Cethosia nietneri nietneri Fd. (Tamil lace wing) $)^{\mathrm{En}_{\mathrm{n}} \mathrm{Th}_{\mathrm{H}}}$

32. Byblia ilithyia Dry: (joker) ${ }^{\mathrm{RTh}}$

33. Ariadine minorata M. (angled Castor)

34. Telchinia violae $\mathrm{F}$. (tawny Castor)

\section{Family Erycinidac}

35. Lepia lepitoides M. (beak Libythea) ${ }^{\mathrm{R} T \mathrm{~T}}$

36. Abisara acherius prunosa M. (plum Judy)

\section{Family Lycaenidac}

37. Spalgis epeus epeus Ed. (apefly)

38. Talicada nyseus nyseus Guer. (red pierrot)

39. Castalius rosimon rosimon $\mathrm{F}$. (common pierrot)

40. Tarucus nara Koll. (striped pierrot) ${ }^{\mathrm{R} .}$. Th

41. Syntarucus plinius F. (zebra bluc)

42. Azanus ubaldus $\mathrm{Cr}$. (bright babul blue) ${ }^{\mathrm{R} T \mathrm{~h}}$

43. Neopithecops zalmora But. (quaker)

44. Everes lacturmus parrhasius F. (Indian cupid)

45. Megisba malaya thwaitesi M. (Malayan)

46. Celastrina puspa felderi Toxopeus. (common hedge blue)

47. (hilades lajus lajus $\mathrm{Cr}$. (lime blue)

48. Zizeeria trochilus putli Koll. (grass jewel)

49. Zizula hylax hylax F. (tiny grass blue)

50. Zizina otis decreta But. (lesser grass blue)

51. Euchrusops cnejus F. (gram blue)

52. Anthene lycaenina Fd. (pointed ciliate blue)

53. Catochrusops straho F. (forget-me-not)

54. Lampides boeticus L. (pea blue) 
55. Jamides bochus bochus $\mathrm{Cr}$. (dark cenulean)

56. Jamides celeno tissama Fr. (common cenulean)

57. Jamides alecto meilichius. Fr. (metallic cerulean)

58. Nacaduba pactolus cevlonica Fr. (large four-line blue) ${ }^{\mathrm{R}}$

59. Nacaduba nabo sidoma Fr. (pale four-line blue) ${ }^{\mathrm{R}}$

60. Nacatuba sinhala Ormiston. (Ceylon six-line blue)

61. Nacaduba nora ardates M. (common line blue)

62. Petrelea dana adima Rh'ePhil. (gingy line blue)

63. (uretis thetis Dry. (Indian sumbeam)

64. Iraota timoleon nicevillei But. (silver streak blue) ${ }^{\mathrm{R}, \mathrm{T}_{2}}$

65. Amblypodia anita naradoides M. (purple leaf blue)

66. Arhopala centaurus pirama M. (centaur oakblue)

67. Surendra quercetorum discalis $\mathrm{M}$. (common acacia blue)

68. Atymmus arcuata M. (yam fly loxura)

69. Spindasis vulcanus fasca M. (common silverline)

70. Zesius chrvsomallus Hub. (redspot)

71. Pratapa deva deva M. (white royal) ${ }^{\mathrm{R} T \mathrm{Th}}$

72. Tajuria jehana M. (plains bluc royal) $)^{\mathrm{RTh}}$

73. Cheritra fieja pseudojaffra M. (common imperial)

74. Rathinda amor F. (monkey puzzle)

75. Horaga onvx cingalensis $\mathrm{M}$. (common onyx) ${ }^{\mathrm{R}}$

76. Catapaecilma major myositina Fr. (common tinsel) ${ }^{\mathrm{Th}_{\mathrm{h}}}$

77. Hypolycaena ni/girica M. (Nilgiri tit) ${ }^{\mathrm{Th}}$

78. Deudorix epijarbas epijarbas M. (comelian)

79. Virachola isocrates $\mathrm{F}$. (guava blue) ${ }^{\mathrm{T}_{2}}$

80. Rapala lankana M. (Malabar flash) ${ }^{V_{\mathrm{r}}, \mathrm{Th}_{\mathrm{t}}}$

81. Phocides moorei Fr. (plane bindahara) ${ }^{\mathrm{Th}}$

\section{Family Pieridae}

82. Leptosia nina nina $\mathrm{F}$. (Psyche)

83. Delias euch aris Dry. (common Jezebel)

84. Prioneris sita $\mathrm{Fd}$. (painted saw-tooth) ${ }^{\mathrm{R}, \mathrm{Th}}$

85. Anapheisaurota taprobana M. (pioneer)

86. Huphina narssa phryne $\mathrm{F}$. (common gull)

87. Appias indra narendra M. (plain puffin) ${ }^{\mathrm{V}_{\mathrm{r}}, \mathrm{Th}_{\mathrm{L}}}$

88. Appiass albina darata $\mathrm{Fd}$. (common albatross)

89. Catopsilia crocale crocale $\mathrm{Cr}$. (common emigrant)

90. Eurema vrigitta rubella Wallace. (small grass yellow)

91. Eurema blanda silhetana Wallace. (tree-spot grass yellow)

92. Eurema hecabe simulata M. (common grass yellow)

93. Lxias marianne $\mathrm{Cr}$. (white orange tip)

94. Colotis calais modestus But. (small salmon Arab)

95. Colotis etrida limbatus But. (little orange tip) ${ }^{\mathrm{En}}$

96. (olotis danae danae F. (crimson tip)

97. Hebomoia glaucippe australis But. (great orange tip)

98. Valeria ceylanica ceylanica $\mathrm{Fd}$. (dark wanderer) 


\section{Family Papilionidac}

99. Troides helena darsius Gray. (common birdwing) ${ }^{\mathrm{E}_{\mathrm{L}}} \mathrm{Th}$ 100. Polvdonus jophon jophon Gray. (Ceylon rose) ${ }^{\mathrm{En}, \mathrm{R}, \mathrm{Th}}$ 101. (' hilasa clytia tankeswara M. (common mime) ${ }^{\mathrm{E} 1}$ 102. Papilio polymnestor parinda $\mathrm{M}$. (The Blue Mormon) ${ }^{\mathrm{En}} \mathrm{M}_{\mathrm{H}}$ 103. (rraphium nomius nomius Esp. (spot swordtail) ${ }^{\mathrm{Th}}$ 104. Papilio crino $\mathrm{F}$. (common banded peacock) ${ }^{\mathrm{T}_{\mathrm{t}}}$ 105. Papilio demoleus demoleus L. (lime butterfly)

\section{Family Hesperiidae}

106. Hasora padra lanka Evans. (Ceylon awl) ${ }^{\mathrm{R} . \mathrm{T}_{\mathrm{L}}}$

107. Bihasis vedipodea ataphus Watson. (branded orange awlet) ${ }^{\mathrm{Th}}$

108. (hoaspes benjaminii benjaminii Guer. (Indian awl king)

109. Badamia exclamationis F. (brown awl)

110. Celaenorrhinus spilothyrus Fd. (black flat)

111. Tagiades gapetus obscurus Mab. (Ceylon snow flat $)^{\text {Ent Th }}$

112. Coladenia indrani tissa M. (tricolour pied flat)

113. Sarangesa dasahara albicilia M. (common small flat)

114. Tapena thwaitesi thwaitesi M. (black angle) ${ }^{\mathrm{R}, \mathrm{Th}}$

115. (aprona ransonnettii ransonnettii $\mathrm{Fd}$. (golden angle) $)^{\text {th }}$

116. (iomalia elma albofasciata $\mathrm{M}$. (African marbled skipper) ${ }^{\mathrm{V}_{\mathrm{r}} \mathrm{Th}}$

117. Spalia galba $\mathrm{F}$. (Indian skipper)

118. Baracus vittatus vittatus Fd. (hedge hopper) ${ }^{\mathrm{En}}$

119. Ampittia dioscorides singa Evans. (bush hopper)

120. Lambrix salsala luteipalpus PI. (chestnut bob)

121. Suastus gremius subgrisea M. (Indian palm bob) ${ }^{\text {Th }}$

122. Udaspes folus $\mathrm{Cr}$. (grass demon) ${ }^{\mathrm{Vir}_{\mathrm{r}} \mathrm{Th}}$

123. Notocrypta paralysos livsia Evans. (common banded demon)

124. Gangara thyrsis clothilda Herrich-Schaffer. (giant redeye) ${ }^{\text {Th }}$

125. Matapa aria $\mathrm{M}$. (common redeye)

126. Hyarotis adrastus adrastus $\mathrm{Cr}$. (tree filter)

127. Halpe decrata $\mathrm{M}$. (decorated ace) ${ }^{\mathrm{E} L \mathrm{R}}$

128. Halpe homolea scylonica M. (Ceylon ace)

129. Taractrocera maevius maevius $F$. (common grass dart)

130. Oriens goloides M. (common dartlet)

131. Potanthus augusta satra Fr. (tropic dart)

132. Telicota colon amba Evans. (pale palm dart)

133. Baoris farri penicillata $\mathrm{M}$. (paint brush swift) ${ }^{\mathrm{R}, \text { Th }}$

134. (altoris kumara lanka Evans. (blank swift)

135. Pelopidas conjuncta narooa $\mathrm{M}$. (conjoined swift)

136. Parnara bada bada M. (smallest swift)

"En = Endemic, $\mathrm{Vr}=$ Very rare, $\mathrm{R}=$ Rare,

$\mathrm{Th}=$ Threatened, $\mathrm{RI}=$ IUCN Red-Book threatened species 